\title{
Autoimmune Connective Tissue Disease Following Carbon Monoxide Poisoning: A Nationwide Population-Based Cohort Study
}

This article was published in the following Dove Press journal: Clinical Epidemiology

\author{
Chien-Cheng Huang (iD) ${ }^{1-3}$ \\ Chung-Han $\mathrm{Ho}$ iD $^{4,5}$ \\ Yi-Chen Chen (iD ${ }^{4}$ \\ Chien-Chin Hsu ${ }^{1,6}$ \\ Hung-Jung Lin ${ }^{1,7}$ \\ Jhi-Joung Wang ${ }^{4,8}$ \\ How-Ran Guo iD $2,9,10$
}

'Department of Emergency Medicine, Chi Mei Medical Center, Tainan, Taiwan; ${ }^{2}$ Department of Environmental and Occupational Health, College of Medicine, National Cheng Kung University, Tainan, Taiwan; ${ }^{3}$ Department of Senior Services, Southern Taiwan University of Science and Technology, Tainan, Taiwan; ${ }^{4}$ Department of Medical Research, Chi Mei Medical Center, Tainan, Taiwan; ${ }^{5}$ Department of Hospital and Health Care Administration, Chia Nan University of Pharmacy and Science, Tainan, Taiwan; ${ }^{6}$ Department of Biotechnology, Southern Taiwan University of Science and Technology, Tainan, Taiwan; ${ }^{7}$ Department of Emergency Medicine, Taipei Medical University, Taipei, Taiwan; ${ }^{8}$ Allied Al Biomed Center, Southern Taiwan University of Science and Technology, Tainan, Taiwan; ${ }^{9}$ Department of Occupational and Environmental Medicine, National Cheng Kung University Hospital, Tainan, Taiwan; ${ }^{10}$ Occupational Safety, Health and Medicine Research Center, National Cheng Kung University Hospital, Tainan, Taiwan

Correspondence: How-Ran Guo Department of Environmental and Occupational Health, College of Medicine, National Cheng Kung University, I Daxue Road, East District, Tainan City 70I, Taiwan

Tel +886-6-235-3535

Fax +886-6-275-2484

Email hrguo@mail.ncku.edu.tw
Background: In addition to hypoxia, oxidative stress and inflammation due to carbon monoxide (CO) poisoning cause adverse health effects. These mechanisms are related to the occurrence of autoimmune connective tissue disease, but studies on the association between $\mathrm{CO}$ poisoning and autoimmune connective tissue disease are limited. We conducted a study to evaluate the occurrence of autoimmune connective tissue disease following $\mathrm{CO}$ poisoning.

Methods: We identified participants with CO poisoning diagnosed between 1999 and 2012 from the Nationwide Poisoning Database and selected participants without CO poisoning from the Taiwan National Health Insurance Research Database with matching age and index dates at a 1:3 ratio. Sex, underlying comorbidities, and monthly income were also included in the analyses. We followed up the participants until 2013 and made comparison of the risk of autoimmune connective tissue disease between participants with and without $\mathrm{CO}$ poisoning. Results: The 23,877 participants with $\mathrm{CO}$ poisoning had a higher risk for autoimmune connective tissue disease than the 71,631 participants without $\mathrm{CO}$ poisoning (adjusted hazard ratio [AHR], 3.5; 95\% confidence interval [CI], 3.1-3.9) after adjustment for sex, diabetes, Lyme disease, herpes zoster, infectious mononucleosis, hepatitis, HIV infection, liver disease, renal disease, non-CO poisoning or drug abuse, malignancy, hypertension, hyperlipidemia, coronary artery disease, congestive heart failure, chronic obstructive pulmonary disease, and monthly income. An increased risk was observed even after 4 years of followup (AHR, 3.6; 95\% CI, 3.0-4.4).

Conclusion: The risk of autoimmune connective tissue disease increased following $\mathrm{CO}$ poisoning. Close follow-up of the patients with CO poisoning for the development of connective tissue disease is recommended, and further investigation of the detailed mechanisms is warranted.

Keywords: autoimmune, carbon monoxide poisoning, connective tissue disease, hypoxia, inflammation, oxidative stress

\section{Introduction}

Carbon monoxide (CO) poisoning is an important health problem worldwide. It accounts for more than 50,000 emergency department visits per year ${ }^{1}$ and 1319 deaths in 2014 in the United States. ${ }^{2}$ In Taiwan, there were 25,912 cases diagnosed between 1999 and 2012 as having CO poisoning, which resulted in about 500 deaths after acute poisoning. ${ }^{3}$ In addition to acute fatality, $\mathrm{CO}$ poisoning also contributed to increased long-term mortality and neurological sequelae including 
impairments of concentration or language, affective behavioral changes, memory loss, inability to calculate, vestibular problem, Parkinsonism, or corticospinal tract signs of damage, etc. ${ }^{3-7}$

A major mechanism through which $\mathrm{CO}$ poisoning introduces adverse health effects is the hypoxic injury to the tissue, which is a result of the nearly 250 times greater affinity that hemoglobin $(\mathrm{Hb})$ has for $\mathrm{CO}$ than oxygen and leftward shift of $\mathrm{O}_{2} \mathrm{Hb}$ dissociation curve. ${ }^{1,8}$ Another mechanism of $\mathrm{CO}$ poisoning is the inflammatory reaction that increases oxidative stress and the production of reactive oxygen species (ROS), which can lead to ongoing inflammation and necrosis. ${ }^{1}$ The main target organs are the heart and brain due to their high need for oxygen. ${ }^{1}$ A study based on a nationwide database in Taiwan found that $\mathrm{CO}$ poisoning results in a nearly 2 -fold increase in the risk of diabetes ${ }^{9}$ and argued that hypoxic injury, as well as inflammatory and immunological reactions in the brain and other organs, including the pancreas, led to the increased risk. ${ }^{9}$

Autoimmune connective tissue disease, which includes systemic lupus erythematosus, rheumatoid arthritis, Sjögren's syndrome, scleroderma (systemic sclerosis; systemic scleroderma), and mixed connective tissue disease, is characterized by spontaneous overactivity of the immune system that results in over-production of antibodies which are found in the circulatory system. ${ }^{10}$ In Taiwan, the prevalence and incidence of autoimmune connective tissue disease were 101.3 per 100,000 populations and 41.3 per 100,000 person-years, respectively. ${ }^{11}$ Patients with autoimmune connective tissue disease had a significant higher mortality and morbidity than the general population. ${ }^{11}$ In addition to genetic factors, environmental factors are found to be associated with autoimmune connective tissue disease. ${ }^{12,13}$ Hypoxia, oxidative stress, and inflammation induced by $\mathrm{CO}$ poisoning may increase the risk of autoimmune connective tissue disease through the production of autoantibodies. ${ }^{14-19}$ Oxidative stress contributes to the pathogenesis, organ damages, and comorbidities in systemic lupus erythematosus. ${ }^{19}$ The effects of oxidative stress include oxidative modification of self-antigens and T-cell dysfunction. ${ }^{19}$ Oxidative stress is also suggested to be associated with mitochondrial dysfunction, which may lead to Sjøgren's syndrome. ${ }^{18}$ There were no reports on the association between $\mathrm{CO}$ poisoning and autoimmune connective tissue disease in a literature search using "carbon monoxide poisoning," "connective tissue disease," “systemic lupus erythematosus," "rheumatoid arthritis," "Sjögren's syndrome," "scleroderma," "systemic sclerosis," and "systemic scleroderma" as keywords through PubMed and Google Scholar. Therefore, we hypothesized that $\mathrm{CO}$ poisoning may be associated with autoimmune connective tissue disease through hypoxic injury, oxidative stress, or both and thus conducted a study to evaluate this potential association.

\section{Materials and Methods Data Sources}

In this nationwide population-based cohort study, we used two subsets of data from the Taiwan National Health Insurance Research Database (NHIRD), namely, the Nationwide Poison Database (NPD) and the Longitudinal Health Insurance Database 2000 (LHID2000). The National Health Research Institutes maintain the NHIRD, which covers nearly $100 \%$ of the population in Taiwan, and provides it to scientists for research purposes. ${ }^{3}$ The NPD includes all the cases of poisonings between 1999 and 2013 in Taiwan, and the LHID2000 contains all the registration and claim data on 1,000,000 individuals randomly selected from the original NHIRD. ${ }^{3}$

\section{Study Design, Setting, and Participants}

We identified all patients diagnosed with $\mathrm{CO}$ poisoning between 1999 and 2012 from the NPD as the study cohort. The comparison cohort was made up of participants without $\mathrm{CO}$ poisoning who were randomly selected from the LHID2000 through exact matching of ages and index dates with the study cohort at a 1:3 ratio. According to our previous studies, ${ }^{20,21}$ the $1: 3$ ratio for matching is sufficient for providing statistical power to answer the research question with adjustment for major potential confounding factors. The index date was defined as the date of hospitalization or visit to the emergency department by the participant with $\mathrm{CO}$ poisoning (Figure 1).

\section{Definitions of Variables}

We defined a patient with $\mathrm{CO}$ poisoning as a participant who has been assigned diagnosis codes 986, E868, E952, or E982 according to the International Classification of Diseases, Ninth Revision, Clinical Modification (ICD9-CM) during hospitalization or a visit to the emergency department. The use of both ICD-9-CM of 986 and E-codes (including E868, E952, and E982) has been validated as an effective method to identify patients with $\mathrm{CO}$ poisoning $^{22}$ and adopted in many studies. . $^{3,7,9,20,21}$ 


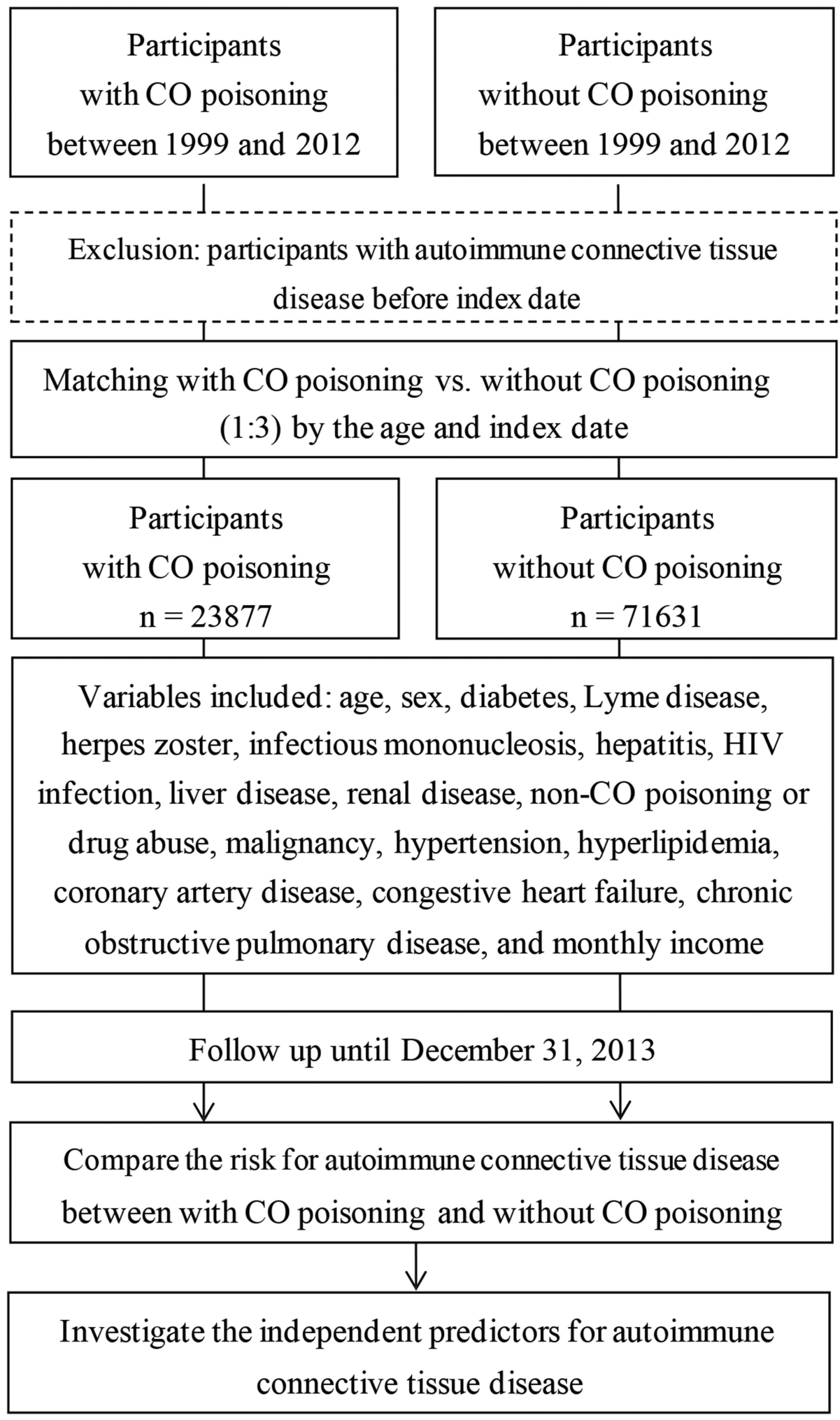

Figure I Flowchart of this study.

Abbreviation: $\mathrm{CO}$, carbon monoxide.

A patient of autoimmune connective tissue disease was defined as a participant who has been assigned ICD-9-CM diagnosis codes 710 or 714 during at least one hospitalization or at least three visits for ambulatory care. Those who had been diagnosed with autoimmune connective tissue disease before the index date were excluded. 
Therefore, all patients with $\mathrm{CO}$ poisoning were right censored on December 31, 2013 or on the date of autoimmune connective tissue disease diagnosis, withdrew from the insurance program, lost for follow-up, or presence of mortality records. The person-years of follow-up were calculated accordingly for each participant, and the maximum follow-up period for participants was 15 years.

The age subgroups were defined as $<20,20-34,35-49$, $50-64$, and $\geq 65$ years. We studied underlying comorbidities and potential confounders for autoimmune connective tissue disease in the analyses, ${ }^{23-25}$ including diabetes (ICD-9-CM: 250), Lyme disease (ICD-9-CM: 088.81), herpes zoster (ICD-9-CM: 053), infectious mononucleosis (ICD-9-CM: 075), hepatitis (ICD-9-CM: 070), HIV infection (ICD9-CM: 042, 07953, or V08), liver disease (ICD-9-CM: 570-576), renal disease (ICD-9-CM: 580-593), non-CO poisoning or drug abuse (ICD-9-CM: 960-989, 303-305), malignancy (ICD-9-CM: 140-208), hypertension (ICD9-CM: 401-405), hyperlipidemia (ICD-9-CM: 272), coronary artery disease (ICD-9-CM: 410-414), congestive heart failure (ICD-9-CM: 428), and chronic obstructive pulmonary disease (ICD-9-CM: 496). Liver disease included acute and subacute necrosis of liver (ICD-9-CM: 570), chronic liver disease and cirrhosis (ICD-9-CM: 571), liver abscess and sequelae of chronic liver disease (ICD-9-CM: 572), other disorders of liver (ICD-9-CM: 573), cholelithiasis (ICD9-CM: 574), other disorders of gallbladder (ICD-9-CM: 575), and other disorders of biliary tract (ICD-9-CM: 576). The subgroups of monthly income were defined as $<20,000$, 20,000-40,000, and >40,000 New Taiwan Dollars (NTD).

\section{Comparison of the Risk of Autoimmune Connective Tissue Disease Between the Two Cohorts}

We compared the risk of autoimmune connective tissue disease between the two cohorts by following up the participants until 2013. Stratified analyses were also performed according to the common types of autoimmune connective tissue disease (systemic lupus erythematosus [ICD-9-CM: 710.0], rheumatoid arthritis [ICD-9-CM: 714], and Sjögren's syndrome and Scleroderma [ICD9-CM: 710.2, 710.1]), age group, sex, underlying comorbidities, and follow-up period.

\section{Ethical Statement}

We conducted this study strictly according to the Declaration of Helsinki. This study was reviewed and approved by the Institutional Review Board (IRB) at Chi Mei Medical Center (approval number: 10407-E01). The NHIRD is not freely available and is limited to research purposes only. Applicants must follow the regulations of National Health Insurance Administration and National Health Research Institutes. All applications are reviewed for approval of data release. Informed consent from the participants was waived by the IRB because the NHIRD contains de-identified information only. The waiver did not affect the rights and welfare of the participants.

\section{Statistical Methods}

In the comparisons of demographic data, underlying comorbidities, and monthly income between the two cohorts, we used independent $t$-tests to evaluate the differences in continuous variables and chi-square tests to evaluate those in categorical variables. The comparison of the risk for autoimmune connective tissue disease between the two cohorts was performed using Cox proportional hazard regression analysis with adjustment for sex, monthly income, and comorbidities. Crude hazard ratio (HR) was defined as the incidence rate of autoimmune connective tissue disease in the participants with $\mathrm{CO}$ poisoning divided by that in the participants without $\mathrm{CO}$ poisoning. Adjusted HR (AHR) was obtained through including potential confounders in the regression model. Because "death" is a competing risk with the outcome measurement of "autoimmune connective tissue disease" in this study, we also performed a competing risk survival analysis to compare with the results by Cox proportional hazard regression analysis. The Kaplan-Meier's method and the Log rank test were used to compare the risk for autoimmune connective tissue disease between the two cohorts during the follow-up. We used Cox proportional hazard regression analysis to identify independent predictors for autoimmune connective tissue disease and evaluate their effects. All the analyses were performed using SAS 9.4 for Windows (SAS Institute, Cary, NC, USA) at a two-tailed significance level of 0.05 .

\section{Results}

In total, we identified 23,877 participants with $\mathrm{CO}$ poisoning and 71,631 participants without $\mathrm{CO}$ poisoning for this study. The mean age was 36.4 (standard deviation=15.5) years, and $39.2 \%$ of participants with $\mathrm{CO}$ poisoning were 20-34 years of age, followed by $31.7 \%$ in $35-49$ years (Table 1). The sex ratio within participants with $\mathrm{CO}$ poisoning was nearly 1 to 1 . There were higher rates of 
Table I Age, Sex, Underlying Comorbidities, and Monthly Income in Participants with and without CO Poisoning

\begin{tabular}{|c|c|c|c|}
\hline Variables & $\begin{array}{l}\text { With CO Poisoning } \\
n=23,877\end{array}$ & $\begin{array}{l}\text { Without CO Poisoning } \\
n=71,631\end{array}$ & $p$-value \\
\hline Age (years) & $36.4 \pm 15.5$ & $36.4 \pm 15.5$ & $>0.999$ \\
\hline \multicolumn{4}{|l|}{ Age (years) } \\
\hline$<20$ & $2680(11.2)$ & $8043(11.2)$ & $>0.999$ \\
\hline $20-34$ & $9366(39.2)$ & $28,096(39.2)$ & \\
\hline $35-49$ & 7566 (31.7) & $22,698(31.7)$ & \\
\hline $50-64$ & $2974(12.5)$ & $8920(12.5)$ & \\
\hline$\geq 65$ & I29| (5.4) & $3874(5.4)$ & \\
\hline \multicolumn{4}{|l|}{ Sex } \\
\hline Female & II,905 (49.9) & $36,878(51.5)$ & $<0.001$ \\
\hline Male & II,972 (50.I) & $34,753(48.5)$ & \\
\hline \multicolumn{4}{|l|}{ Underlying comorbidity } \\
\hline Diabetes & $1439(6.0)$ & $3460(4.8)$ & $<0.001$ \\
\hline Alcohol abuse & $873(3.7)$ & $661(0.9)$ & $<0.001$ \\
\hline Lyme disease & $0(0)$ & $3(<0.1)$ & 0.317 \\
\hline Herpes zoster & $274(1.2)$ & $876(1.2)$ & 0.355 \\
\hline Infectious mononucleosis & $6(<0.1)$ & $22(<0.1)$ & 0.663 \\
\hline Hepatitis & $55(0.2)$ & $2185(3.1)$ & $<0.001$ \\
\hline HIV infection & $64(0.3)$ & $54(0.1)$ & $<0.001$ \\
\hline Liver disease & $3272(13.7)$ & $8372(11.7)$ & $<0.001$ \\
\hline Renal disease & $2336(9.8)$ & $5120(7.2)$ & $<0.001$ \\
\hline Non-CO poisoning or drug abuse & $2122(8.9)$ & $1064(1.5)$ & $<0.001$ \\
\hline Malignancy & $596(2.5)$ & $1426(2.0)$ & $<0.001$ \\
\hline Hypertension & $2730(11.4)$ & $7404(10.3)$ & $<0.001$ \\
\hline Hyperlipidemia & $1893(7.9)$ & $5168(7.2)$ & $<0.001$ \\
\hline Coronary artery disease & $1289(5.4)$ & $2931(4.1)$ & $<0.001$ \\
\hline Congestive heart failure & $343(1.4)$ & $690(1.0)$ & $<0.001$ \\
\hline Chronic obstructive pulmonary disease & $362(1.5)$ & $723(1.0)$ & $<0.001$ \\
\hline \multicolumn{4}{|l|}{ Monthly income (NTD) } \\
\hline$<19,999$ & $17,273(72.3)$ & $44,649(62.3)$ & $<0.001$ \\
\hline $20,000-39,999$ & $5292(22.2)$ & $19,929(27.8)$ & \\
\hline$\geq 40,000$ & $1312(5.5)$ & $7053(9.9)$ & \\
\hline
\end{tabular}

Note: Data are expressed as mean \pm SD or $n(\%)$.

Abbreviations: CO, carbon monoxide; NTD, new Taiwan dollars.

prevalence of many underlying comorbidities in the participants with $\mathrm{CO}$ poisoning than in the participants without CO poisoning, including diabetes, alcohol abuse, HIV infection, liver disease, renal disease, non-CO poisoning or drug abuse, malignancy, hypertension, hyperlipidemia, coronary artery disease, congestive heart failure, and chronic obstructive pulmonary disease. However, participants with $\mathrm{CO}$ poisoning had a lower prevalence rate of hepatitis. Participants with $\mathrm{CO}$ poisoning also had a higher percentage of earning low monthly income than the participants without $\mathrm{CO}$ poisoning.

Compared to participants without $\mathrm{CO}$ poisoning, participants with CO poisoning had an increased risk for autoimmune connective tissue disease after adjusting for sex, diabetes, Lyme disease, herpes zoster, infectious mononucleosis, hepatitis, HIV infection, liver disease, renal 


\begin{tabular}{|c|c|c|c|c|c|c|c|c|c|c|c|c|c|c|c|}
\hline \multicolumn{2}{|c|}{ 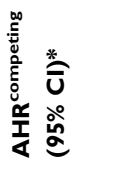 } & 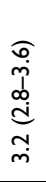 & & & 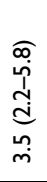 & $\begin{array}{l}\underset{c}{m} \\
\stackrel{b}{d} \\
\bar{d} \\
\bar{m}\end{array}$ & $\begin{array}{l}\widetilde{F} \\
\dot{j} \\
\dot{d} \\
\stackrel{i}{j}\end{array}$ & 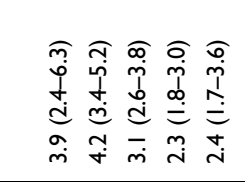 & 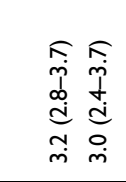 & 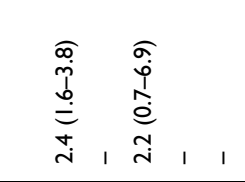 & 1 & 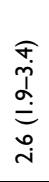 & 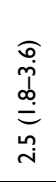 & 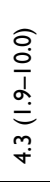 & 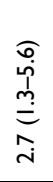 \\
\hline \multicolumn{2}{|c|}{ 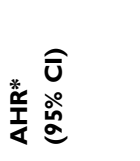 } & $\begin{array}{c}\underset{\sigma}{p} \\
\frac{1}{m} \\
\substack{n \\
m} \\
m\end{array}$ & & & 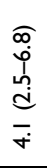 & 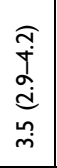 & $\begin{array}{l}\sigma \\
\dot{j} \\
\hat{d} \\
m \\
m \\
m\end{array}$ & 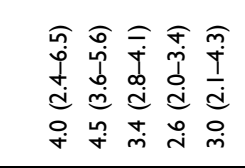 & 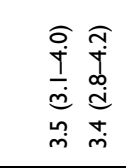 & 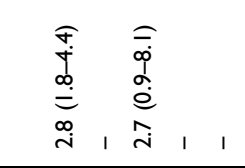 & 1 & $\begin{array}{l}a \\
\dot{1} \\
\stackrel{1}{d} \\
\text { à } \\
\text { ì }\end{array}$ & $\begin{array}{l}\widehat{\bar{j}} \\
\stackrel{d}{d} \\
\stackrel{i}{\dot{i}}\end{array}$ & 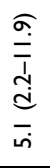 & 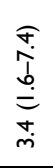 \\
\hline \multicolumn{2}{|c|}{ 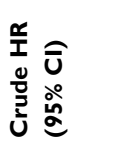 } & 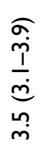 & & & $\begin{array}{l}\text { א. } \\
\dot{L} \\
\stackrel{d}{d} \\
\dot{\sigma}\end{array}$ & 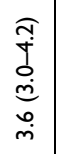 & $\begin{array}{l}\widehat{\infty} \\
\stackrel{i}{0} \\
\stackrel{d}{j} \\
\sim \\
m\end{array}$ & 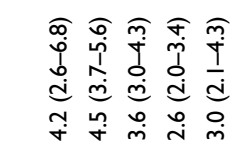 & 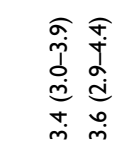 & 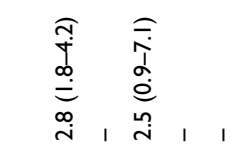 & 1 & $\begin{array}{l}\widehat{y} \\
\underset{j}{d} \\
\stackrel{j}{m}\end{array}$ & 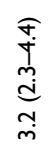 & 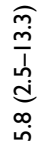 & 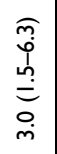 \\
\hline \multirow[b]{3}{*}{ 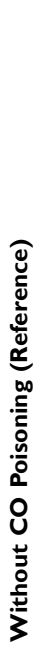 } & 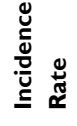 & $\stackrel{\circ}{-}$ & & & $\overline{0}$ & $\hat{o}$ & $\hat{0}$ & 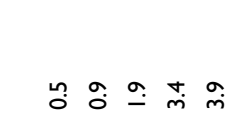 & స̃ $\stackrel{-}{-}$ & $\stackrel{\sim}{m}, \stackrel{\infty}{\sim}, \stackrel{m}{\sim}$ & นึ & $\stackrel{\infty}{i}$ & $\stackrel{\infty}{i}$ & $\underline{\underline{n}}$ & $\hat{i}$ \\
\hline & ฉ & 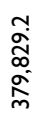 & & & 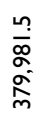 & 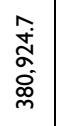 & 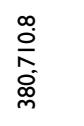 & 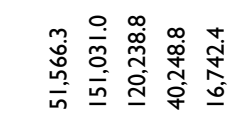 & 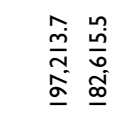 & 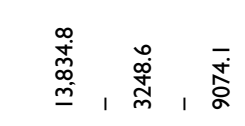 & 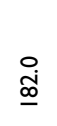 & $\begin{array}{l}\overline{0} \\
\hat{s} \\
\text { o }\end{array}$ & 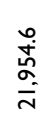 & $\hat{\widetilde{\sigma}}$ & $\stackrel{\text { กิ }}{\mathrm{H}}$ \\
\hline & 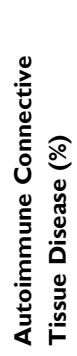 & 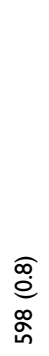 & & & $\begin{array}{l}\widehat{\hat{P}} \\
\text { in } \\
0\end{array}$ & 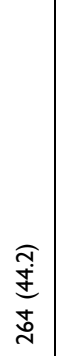 & $\begin{array}{l}\widehat{\widehat{m}} \\
\frac{\dot{y}}{\tilde{d}}\end{array}$ & 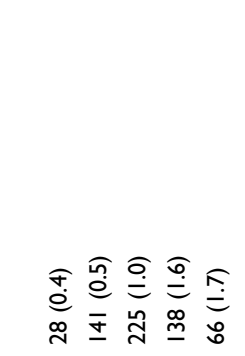 & 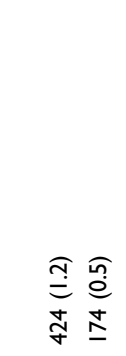 & 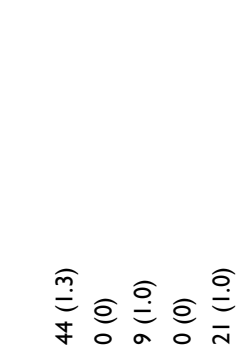 & $\stackrel{\sigma}{\stackrel{\sigma}{E}}$ & 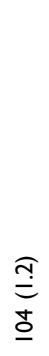 & $\frac{\widehat{T}}{\stackrel{T}{\Xi}}$ & $\underset{0}{\stackrel{0}{0}}$ & 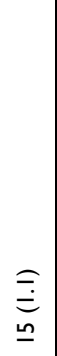 \\
\hline \multirow[b]{3}{*}{ 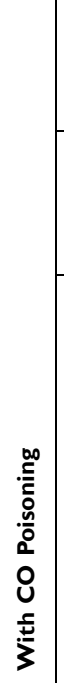 } & 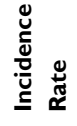 & นึ. & & & $\stackrel{m}{o}$ & $\stackrel{+}{\sim}$ & $\stackrel{\circ}{i}$ & 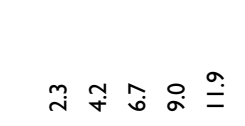 & $\stackrel{+}{\stackrel{亠}{m}}$ & $\underset{\infty}{\infty}, \underset{\sim}{\mathbb{N}}, 1$ & 1 & $\stackrel{\circ}{\circ}$ & $\begin{array}{l}\infty \\
\infty \\
\infty\end{array}$ & $\stackrel{\sim}{\infty}$ & $\underset{\infty}{m}$ \\
\hline & $\grave{2}$ & 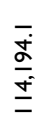 & & & 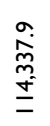 & 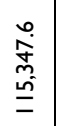 & 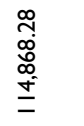 & 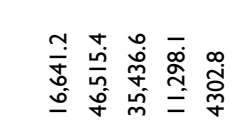 & 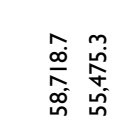 & 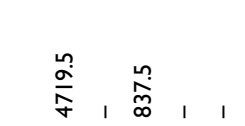 & 1 & 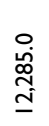 & 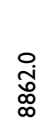 & 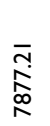 & 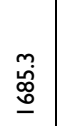 \\
\hline & 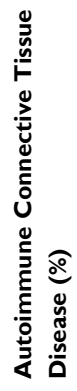 & 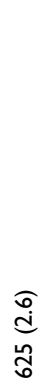 & & & 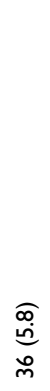 & 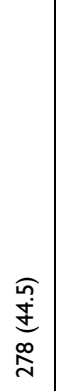 & $\begin{array}{l}\widehat{0} \\
\stackrel{0}{0} \\
\text { aे }\end{array}$ & 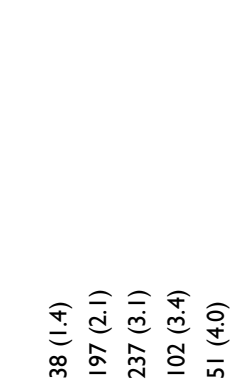 & 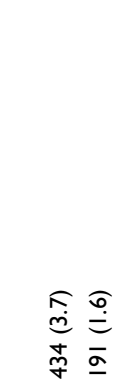 & 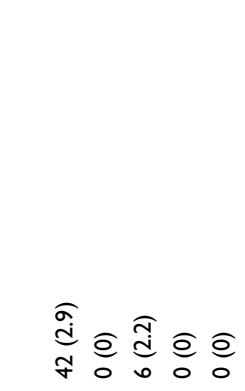 & $\widehat{o}$ & $\begin{array}{l}\widehat{\widehat{F}} \\
\stackrel{e}{c} \\
\equiv\end{array}$ & $\begin{array}{l}\widehat{m} \\
\stackrel{m}{0} \\
\infty\end{array}$ & 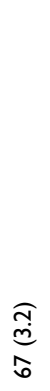 & $\begin{array}{l}\underset{d}{d} \\
\text { d }\end{array}$ \\
\hline \multicolumn{2}{|l|}{ 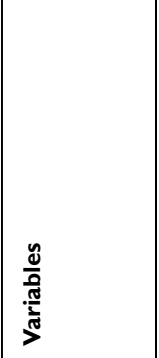 } & 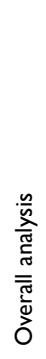 & 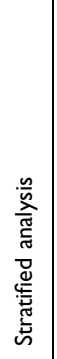 & 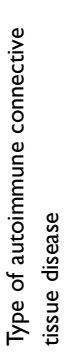 & 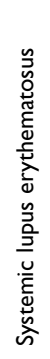 & 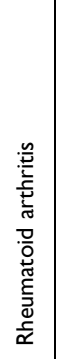 & 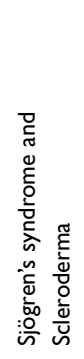 & 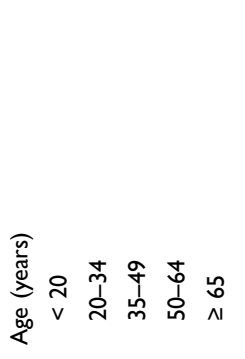 & 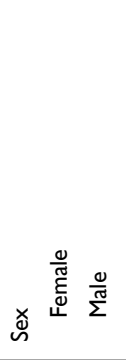 & 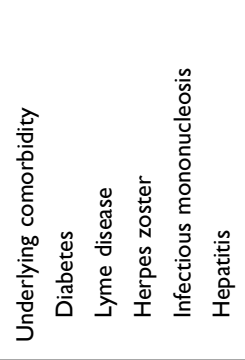 & 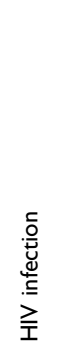 & 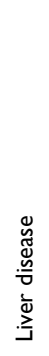 & 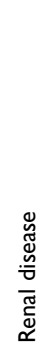 & 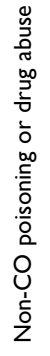 & 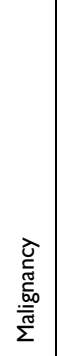 \\
\hline
\end{tabular}




\begin{tabular}{|c|c|c|c|c|c|}
\hline 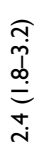 & 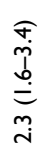 & 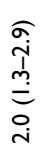 & 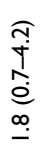 & 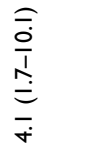 & 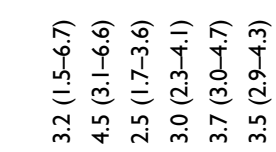 \\
\hline $\begin{array}{l}\underset{r}{p} \\
\frac{1}{d} \\
\substack{\infty \\
i}\end{array}$ & 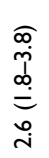 & 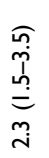 & 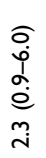 & $\begin{array}{l}\frac{\mathfrak{d}}{m} \\
\frac{1}{d} \\
\dot{d} \\
m \\
\dot{n}\end{array}$ & 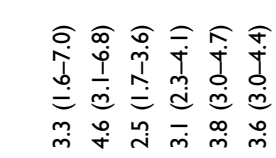 \\
\hline 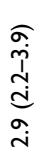 & 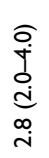 & 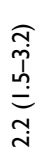 & 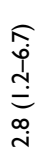 & 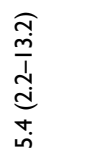 & 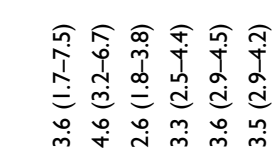 \\
\hline$\stackrel{m}{m}$ & $\stackrel{m}{m}$ & $\stackrel{\infty}{+}$ & $\bar{r}$ & $\stackrel{+}{i}$ & 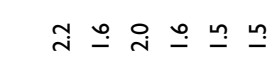 \\
\hline$\frac{\infty}{\stackrel{\infty}{c}}$ & 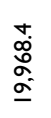 & $\begin{array}{l}\stackrel{+}{\circ} \\
\stackrel{\circ}{=} \\
=\end{array}$ & $\underset{\substack{j \\
\text { d }}}{\stackrel{d}{N}}$ & 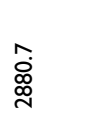 & 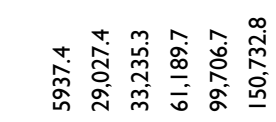 \\
\hline 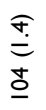 & 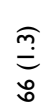 & $\underset{\text { in }}{\stackrel{\sigma}{=}}$ & 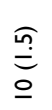 & $\underset{\sim}{\stackrel{\sigma}{\Xi}}$ & 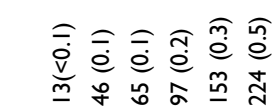 \\
\hline$\hat{\sigma}$ & $\stackrel{t}{\sigma}$ & $\stackrel{ \pm}{\stackrel{0}{0}}$ & 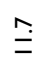 & $\stackrel{m}{\underline{m}}$ & $\stackrel{\infty}{\sim} \stackrel{m}{\sim} \bar{i}$ ஸூ \\
\hline $\begin{array}{l}\stackrel{\sigma}{\tilde{j}} \\
\dot{\sigma}\end{array}$ & 今̊ & $\begin{array}{l}\underset{m}{\tilde{z}} \\
\stackrel{\sim}{y}\end{array}$ & $\overline{\overline{\dot{J}}}$ & ֶָ & 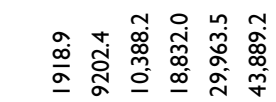 \\
\hline 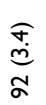 & $\begin{array}{l}\text { त̂ } \\
\tilde{n} \\
0 \\
0\end{array}$ & $\begin{array}{l}\hat{r} \\
\stackrel{n}{2} \\
\hat{f}\end{array}$ & 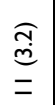 & $\underset{\substack{\overline{\dot{x}} \\
\underline{\underline{n}}}}{c}$ & 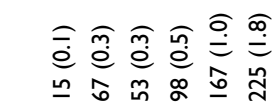 \\
\hline 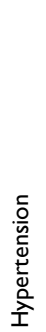 & 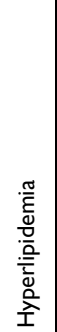 & 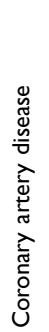 & 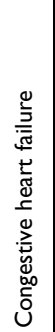 & 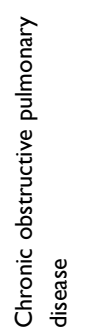 & 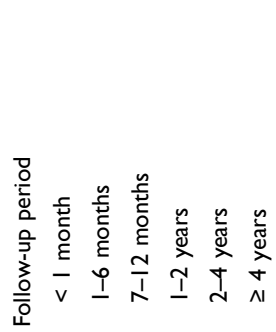 \\
\hline
\end{tabular}

disease, non-CO poisoning or drug abuse, malignancy, hypertension, hyperlipidemia, coronary artery disease, congestive heart failure, chronic obstructive pulmonary disease, and monthly income (AHR, 3.5; 95\% confidence interval [CI], 3.1-3.9) (Table 2). Competing risk survival analysis showed the similar finding (AHR ${ }^{\text {competing }}$, 3.2; 95\% CI, 2.8-3.6). The results were similar in the subgroup analyses for systemic lupus erythematosus, rheumatoid arthritis, and Sjögren's syndrome and scleroderma. Both Kaplan-Meier's method and Log rank test showed an increased risk for autoimmune connective tissue disease in the participants with $\mathrm{CO}$ poisoning compared to in the participants without $\mathrm{CO}$ poisoning (Figure 2). Stratified analyses showed that the increased risk was more prominent in the subgroups with non-CO poisoning or drug abuse (AHR, 5.1; 95\% CI, 2.2-11.9) and the subgroups with chronic obstructive pulmonary disease (AHR, 5.3; 95\% CI, 2.1-13.4). An increased risk was observed during the whole follow-up period, even after 4 years (AHR, 3.6; 95\% CI, 3.0-4.4). We took all the participants including study and comparison cohorts together for analysis and found that $\mathrm{CO}$ poisoning, older age, female sex, liver disease, renal disease, and coronary artery disease were independent predictors for autoimmune connective tissue disease (Table 3).

\section{Discussion}

This study showed that participants with $\mathrm{CO}$ poisoning had an increased risk of autoimmune connective tissue disease than the participants without $\mathrm{CO}$ poisoning, including those who had systemic lupus erythematosus, rheumatoid arthritis, or Sjögren's syndrome and scleroderma and that the increase was observed during the whole follow-up period, even after 4 years. The increased risk was more prominent in participants with chronic obstructive pulmonary disease and participants with non$\mathrm{CO}$ poisoning or drug abuse. In addition to $\mathrm{CO}$ poisoning, older age, female sex, liver disease, renal disease, and coronary artery disease were also identified as independent predictors for autoimmune connective tissue disease.

Connective tissue disease is complex and includes various genetic and environmental factors. ${ }^{13}$ A possible explanation for increased autoimmune connective tissue disease is the hypoxia and oxidative stress induced by CO poisoning. ${ }^{13-17}$ The pathogenesis of autoimmune oxidative stress, a kind of environment factor, is the excess production of ROS and reactive nitrogen species. ${ }^{26-28}$ Excessive oxidative stress may enhance inflammation, 
break down the immunological tolerance, and induce apoptotic cell death, gene activation and generation of novel autoantigens, which are thought to play important roles in the development of autoimmune connective tissue disease. $^{13,28}$ Hypoxia leads to higher collagen synthesis, myofibroblast differentiation of fibroblasts, and productions of cytokine, chemokine, and TGF- $\beta$ by dendritic cells, which all induce the autoimmune reaction. ${ }^{17}$ There is no direct evidence of the association between hypoxia and autoimmune connective tissue disease. However, a study reported that obstructive sleep apnea may increase disease severity and circulating inflammatory markers in patients with autoimmune connective tissue disease. ${ }^{29}$ Many environmental factors, including infections, UV irradiation, coldness, and emotional stress, have been well recognized as developing and exacerbating factors for autoimmune connective tissue disease. ${ }^{13}$ This study showed an association between $\mathrm{CO}$ poisoning and subsequent diagnosis of autoimmune connective tissue disease, which is a novel finding and worthy of further investigation.

Previous studies showed that delayed neuropsychiatric sequelae, a sequela following $\mathrm{CO}$ poisoning, may be caused by immunological response. ${ }^{30,31}$ In rats, CO poisoning causes adduct formation between myelin basic protein (MBP) and malondialdehyde, which results in an immunological cascade. ${ }^{30}$ In the brain tissues of rats with CO poisoning, a variety of microglia and expression of immune factors, including major histocompatibility complex II (MHCII), CD4, vascular cell adhesion molecule-1 (VCAM-1), and interferon-gamma (IFN-gamma) were found. $^{31}$

This study also found that the increased risk for autoimmune connective tissue disease was more prominent in the participants with chronic obstructive pulmonary disease. Smoking is the major causative factor of chronic obstructive pulmonary disease $\mathrm{e}^{32}$ and some studies have shown that carbon monoxide from smoking, an environmental factor, increased the risk for systemic lupus erythematosus. $^{12,33}$ Carbon monoxide and other toxic components from smoking (including tars, polycyclic aromatic hydrocarbons, nicotine, and free radicals) can induce oxidative stress and directly damage endogenous proteins and DNA, leading to genetic mutations and gene activation, which could be involved in development of systemic lupus erythematosus. ${ }^{33}$ Via the same pathophysiology pathway, smoking is also implicated as one of the most important extrinsic risk factors for the development and consequent severity of rheumatoid arthritis. ${ }^{34}$ The association between smoking and rheumatoid arthritis has been demonstrated through epidemiologic studies, in vivo experiments, and animal models. ${ }^{34}$ In patients with primary Sjögren's syndrome, smoking also has a positive association with anti-nuclear antibody positivity. ${ }^{35}$ Smoking is suggested to have negative effects on the vascular, gastrointestinal, and respiratory outcomes of scleroderma. ${ }^{36}$ A possible explanation for the prominent risk in the participants with chronic obstructive pulmonary disease is that smoking, in conjunction with $\mathrm{CO}$ poisoning, might have an additional effect on the development of autoimmune connective tissue disease.

The association between non-CO poisoning or drug abuse and autoimmune connective tissue disease has not been determined. Some studies have found that recreational drugs such as cocaine, amphetamines, marijuana, and heroin, acting as environmental toxins, may stress and injury immune stem cells and engender subsequent autoimmune disease development and progression. ${ }^{37,38}$

Older age and female sex were independent predictors for autoimmune connective tissue disease in this study. Using participants $<20$ years old as references, we found the risk of autoimmune connective tissue disease increased with age $(\mathrm{AHR}=1.7$ in age $20-34$ years, $\mathrm{AHR}=2.9$ in age 35-49 years, AHR $=4.3$ in age 50-64 years, and AHR $=5.0$ in age $\geq 65$ years). Therefore, older age appeared to be a predictor for autoimmune connective tissue disease. In systemic lupus erythematosus, peak incidence occurs between the age of 15 and 40 years old, with a female-tomale ratio of $6: 1$ to $10: 1{ }^{39}$ In a study of the geriatric population, the prevalence of rheumatoid arthritis predominated in participants of the female sex, increased together with age, and reached approximately $2 \%{ }^{40}$ Sjögren's syndrome increases with age and dominates in the female sex with a female-to-male ratio of 9:1. ${ }^{39}$ The onset of scleroderma is most common in those $30-50$ years old, and the female-to-male ratio is between $5: 1$ and $14: 1^{39}$

It is well known that complications of the liver, kidneys, and coronary artery may develop in autoimmune connective tissue disease. ${ }^{41-43}$ In the current study, however, we found that liver disease, renal disease, and coronary artery disease were independent predictors for autoimmune connective tissue disease, which is has not been reported in the literature. A possible explanation is that these complications were sometimes recognized before the diagnosis of autoimmune connective tissue disease. It is also possible that there are 


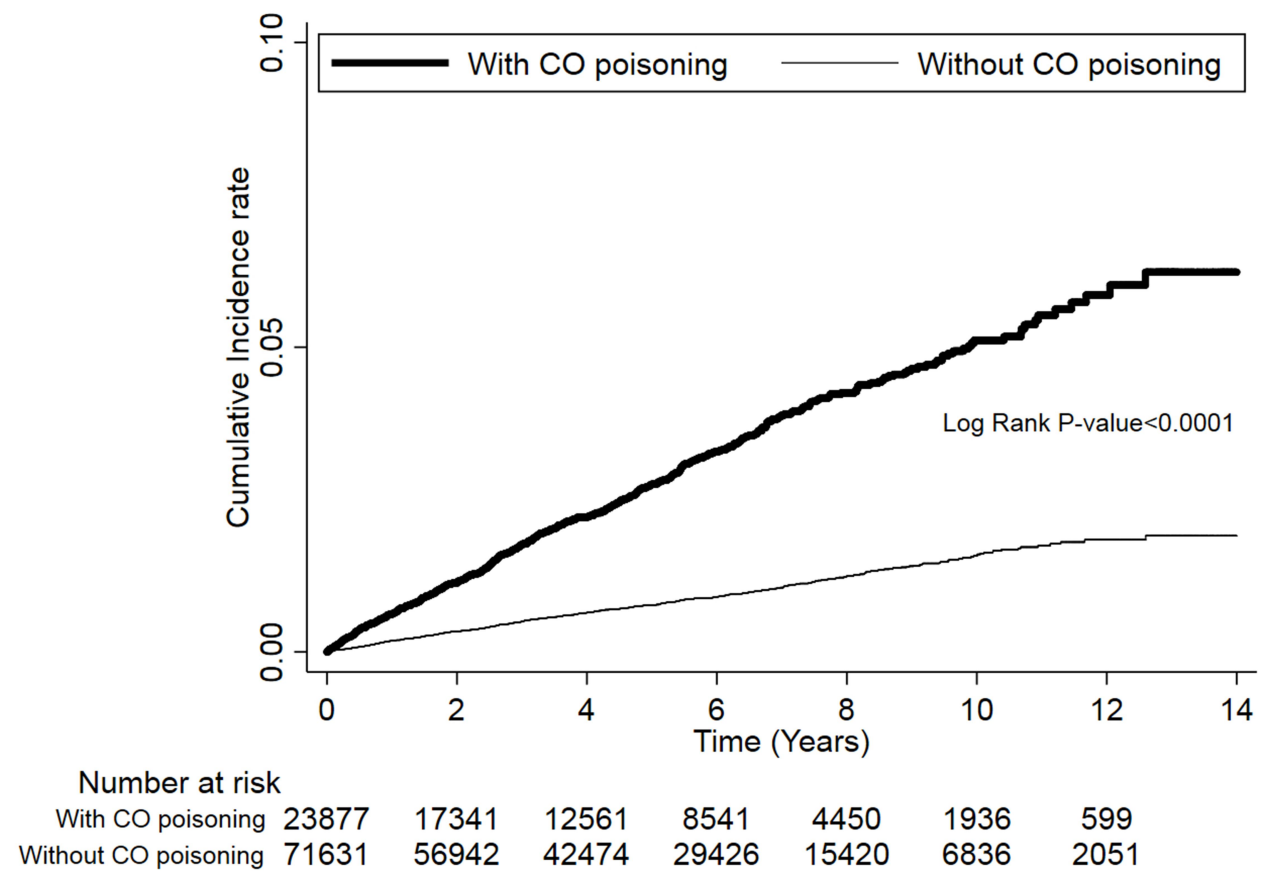

Figure 2 Comparison of the risk of autoimmune connective tissue disease between participants with and without CO poisoning during follow-up by Kaplan-Meier's method and the Log rank test.

Abbreviation: $\mathrm{CO}$, carbon monoxide.

bi-directional causal relationships between these diseases and autoimmune connective tissue disease.

The strength of this study is its novel finding of a higher risk of autoimmune connective tissue disease in patients with $\mathrm{CO}$ poisoning. It provides an insight into a previously unknown relationship between $\mathrm{CO}$ poisoning and autoimmune diseases. In addition to having the potential of informing public health practices, it may also help understanding the contributions of environmental risk factors to autoimmune diseases. It also has some limitations, however. First, the claim data used in this study is not collected for the purposes of research. Validation of definitions for $\mathrm{CO}$ poisoning, connective tissue disease, and underlying comorbidities are needed. Second, the participants without $\mathrm{CO}$ poisoning might have CO poisoning before 1999. Because the NPD includes the data between 1999 and 2013, we could not exclude the participants who had $\mathrm{CO}$ poisoning in the comparison cohort. In addition, some participants with $\mathrm{CO}$ poisoning might not be recognized or diagnosed due to insignificant symptoms and thus selected as members of the comparison cohort. This will lead to underestimation of the effect. However, this study showed an increased risk for autoimmune connective tissue disease in the study cohort using the comparison cohort as the reference and so the conclusion would not be affected by such misclassification.
Third, there were no data on family history, smoking, lifestyle, or environmental exposure in this study, all of which are factors with the potential of confounding the results. However, we have adjusted for common underlying comorbidities, and they might serve as the surrogates for some of the unavailable data. For example, chronic obstructive pulmonary disease might serve as a surrogate measurement of smoking and diabetes, hypertension, and hyperlipidemia might serve as surrogate measurements of lifestyle. Fourth, we did not evaluate the effects of the severity of $\mathrm{CO}$ poisoning on risk for autoimmune connective tissue disease. Further studies which are able to determine the severity of $\mathrm{CO}$ poisoning in the participants are warranted. Fifth, although we included all four major autoimmune connective tissue diseases (systemic lupus erythematosus, rheumatoid arthritis, Sjögren's syndrome, and scleroderma) as the outcomes in the analysis, the results may not stand for all the autoimmune connective tissue diseases. Sixth, despite this being a nationwide study that covered a large population, the result may not be generalized to other nations with different genetic makeups. Validation of the result in other nations is needed. Seventh, this study reported an epidemiological result based on the rationale of hypoxia and oxidative stress; however, further basic studies are needed to clarify the detailed pathophysiology. 
Table 3 Independent Predictors for Autoimmune Connective Tissue Disease in the All Participants by Cox Proportional Hazard Regression Analysis

\begin{tabular}{|c|c|c|c|}
\hline Variables & $\begin{array}{l}\text { Crude HR } \\
(95 \% \mathrm{Cl})\end{array}$ & $\begin{array}{l}\text { AHR } \\
(95 \% \mathrm{Cl}) *\end{array}$ & $p$-value $f$ \\
\hline \multicolumn{4}{|l|}{ Cohort } \\
\hline With CO poisoning & $3.5(3.1-3.9)$ & $3.5(3.1-3.9)$ & $<0.001$ \\
\hline Without $\mathrm{CO}$ poisoning & I (reference) & I (reference) & - \\
\hline \multicolumn{4}{|l|}{ Age (years) } \\
\hline$<20$ & I (reference) & I (reference) & - \\
\hline $20-34$ & $1.8(1.4-2.3)$ & $1.7(1.3-2.2)$ & $<0.001$ \\
\hline $35-49$ & $3.1(2.4-3.9)$ & $2.9(2.2-3.7)$ & $<0.001$ \\
\hline $50-64$ & $4.8(3.6-6.3)$ & $4.3(3.3-5.7)$ & $<0.001$ \\
\hline$\geq 65$ & $5.7(4.2-7.7)$ & $5.0(3.6-7.0)$ & $<0.001$ \\
\hline \multicolumn{4}{|l|}{ Sex } \\
\hline Female & $2(2-2.5)$ & $2.5(2-2.5)$ & $<0.001$ \\
\hline Male & I (reference) & I (reference) & - \\
\hline \multicolumn{4}{|l|}{ Underlying comorbidity } \\
\hline Diabetes & $1.9(1.5-2.4)$ & $0.9(0.7-1.2)$ & 0.441 \\
\hline Lyme disease & - & - & - \\
\hline Herpes zoster & $1.5(0.9-2.4)$ & $\mathrm{I} .0(0.6-1.7)$ & 0.948 \\
\hline Infectious mononucleosis & - & - & - \\
\hline Hepatitis & $0.9(0.6-1.4)$ & $1.0(0.6-1.5)$ & 0.832 \\
\hline HIV infection & I.I (0.2-7.5) & I.I $(0.2-7.5)$ & 0.965 \\
\hline Liver disease & $1.9(1.7-2.2)$ & $1.4(1.2-1.6)$ & $<0.001$ \\
\hline Renal disease & $1.9(1.6-2.3)$ & $1.2(1.0-1.5)$ & 0.032 \\
\hline Non-CO poisoning or drug abuse & $2.5(2.0-3.2)$ & $1.3(1.0-1.6)$ & 0.063 \\
\hline Malignancy & $1.6(1.1-2.3)$ & $0.9(0.6-1.3)$ & 0.655 \\
\hline Hypertension & $2.1(1.8-2.4)$ & I.0 (0.9-I.3) & 0.727 \\
\hline Hyperlipidemia & $2.0(1.7-2.4)$ & I.I (0.9-I.3) & 0.566 \\
\hline Coronary artery disease & $2.7(2.2-3.2)$ & $1.3(1.0-1.6)$ & 0.047 \\
\hline Congestive heart failure & $2.5(1.6-3.8)$ & $1.0(0.6-1.6)$ & 0.973 \\
\hline Chronic obstructive pulmonary disease & $2.2(1.4-3.4)$ & I.I (0.7-I.6) & 0.815 \\
\hline \multicolumn{4}{|l|}{ Monthly income (NTD) } \\
\hline$<19,999$ & $1.2(1.0-1.5)$ & $0.9(0.7-1.1)$ & 0.396 \\
\hline $20,000-39,999$ & $1.0(0.8-1.3)$ & $0.9(0.7-1.2)$ & 0.468 \\
\hline$\geq 40,000$ & I (reference) & I (reference) & - \\
\hline
\end{tabular}

Notes: *Adjusted for age, sex, diabetes, Lyme disease, herpes zoster, infectious mononucleosis, hepatitis, HIV infection, liver disease, renal disease, non-CO poisoning or drug abuse, malignancy, hypertension, hyperlipidemia, coronary artery disease, congestive heart failure, chronic obstructive pulmonary disease, and monthly income. $\dagger$ For AHR.

Abbreviations: AHR, adjusted hazard ratio; $\mathrm{CO}$, carbon monoxide; $\mathrm{Cl}$, confidence interval; ARF, acute respiratory failure; HBOT, hyperbaric oxygen therapy; NTD, new Taiwan dollars.

\section{Conclusions}

This nationwide population-based cohort study found that the risk for autoimmune connective tissue disease increased following $\mathrm{CO}$ poisoning, even in the subgroup's analyses for systemic lupus erythematosus, rheumatoid arthritis, and Sjögren's syndrome and scleroderma. The increase in the risk was observed throughout the whole follow-up period, even after 4 years. Hypoxia, oxidative 
stress, and inflammation are suspected to be the causes. In addition to $\mathrm{CO}$ poisoning, we also identified older age, female sex, liver disease, renal disease, and coronary artery disease as in dependent predictors for autoimmune connective tissue disease. The positive association between smoking and autoimmune connective tissue disease may explain the increased risk of being more prominent in the subgroup with chronic obstructive pulmonary disease. Further studies, including validation in other nations and those that delineate the detailed pathophysiology of the development of autoimmune connective tissue disease as caused by hypoxia and oxidative stress are warranted.

\section{Acknowledgments}

This study was supported by grants MOST 109-2314-B384-005-MY3 and MOST 108-2638-B-006-001-MY2 from the Ministry of Science and Technology and grants CMFHR10734 and CMFHR10832 from Chi Mei Medical Center. We thank Miss Ti Hsu for the English revision.

\section{Author Contributions}

All authors made substantial contributions to conception and design, acquisition of data, or analysis and interpretation of data; took part in drafting the article or revising it critically for important intellectual content; agreed to submit to the current journal; gave final approval of the version to be published; and agree to be accountable for all aspects of the work.

\section{Disclosure}

The authors report no conflicts of interest in this work.

\section{References}

1. Weaver LK. Clinical practice. Carbon monoxide poisoning. $N$ Engl $J$ Med. 2009;360(12):1217-1225. doi:10.1056/NEJMcp0808891

2. Hampson NB. U.S. mortality due to carbon monoxide poisoning, 1999-2014. Accidental and intentional deaths. Ann Am Thorac Soc. 2016;13:1768-1774.

3. Huang $\mathrm{CC}$, Ho $\mathrm{CH}$, Chen $\mathrm{YC}$, et al. Demographic and clinical characteristics of carbon monoxide poisoning: nationwide data between 1999 and 2012 in Taiwan. Scand J Trauma Resusc Emerg Med. 2017;25:70. doi:10.1186/s13049-017-0416-7

4. Huang CC, Chung MH, Weng SF, et al. Long-term prognosis of patients with carbon monoxide poisoning: a nationwide cohort study. PLoS One. 2014;9:e105503. doi:10.1371/journal.pone.0105503

5. Kao YZ, Tsai JF, Chen KT, et al. Carbon monoxide poisoning in geriatric patients. Taiwan Geriatr Gerontol. 2014;9:84-95.

6. Zou JF, Guo Q, Shao H, et al. Lack of pupil reflex and loss of consciousness predict 30-day neurological sequelae in patients with carbon monoxide poisoning. PLoS One. 2015;10(3):e0119126. doi:10.1371/journal.pone.0119126
7. Huang $\mathrm{CC}$, Ho $\mathrm{CH}$, Chen $\mathrm{YC}$, et al. Hyperbaric oxygen therapy is associated with lower short- and long-term mortality in patients with carbon monoxide poisoning. Chest. 2017;152(5):943-953. doi:10.1016/j.chest.2017.03.049

8. Buckley NA, Juurlink DN, Isbister G, Bennett MH, Lavonas EJ. Hyperbaric oxygen for carbon monoxide poisoning. Cochrane Database Syst Rev. 2011;CD002041.

9. Huang -C-C, Ho C-H, Chen Y-C, et al. Increased risk for diabetes mellitus in patients with carbon monoxide poisoning. Oncotarget. 2017;8(38):63680-63690. doi:10.18632/oncotarget.18887

10. Didier K, Bolko L, Giusti D, et al. Autoantibodies associated with connective tissue diseases: what meaning for clinicians? Front Immunol. 2018;9:541

11. Yu KH, See LC, Kuo CF, Chou IJ, Chou MJ. Prevalence and incidence in patients with autoimmune rheumatic diseases: a nationwide population-based study in Taiwan. Arthritis Care Res (Hoboken). 2013;65:244-250. doi:10.1002/acr.21820

12. Barbhaiya M, Costenbader KH. Environmental exposures and the development of systemic lupus erythematosus. Curr Opin Rheumatol. 2016;28:497-505. doi:10.1097/BOR.0000000000000318

13. Kumagai S, Jikimoto T, Saegusa J. [Pathological roles of oxidative stress in autoimmune diseases]. Rinsho Byori. 2003;51:126-132. Japanese.

14. Vona R, Giovannetti A, Gambardella L, Malorni W, Pietraforte D, Straface E. Oxidative stress in the pathogenesis of systemic scleroderma: an overview. J Cell Mol Med. 2018;22(7):3308-3314. doi:10.1111/jcmm. 13630

15. Desai KM, Chang T, Wang $\mathrm{H}$, et al. Oxidative stress and aging: is methylglyoxal the hidden enemy? Can J Physiol Pharmacol. 2010;88:273-284. doi:10.1139/Y10-001

16. Quinonez-Flores CM, Gonzalez-Chavez SA, Del Rio Najera D, Pacheco-Tena C. Oxidative stress relevance in the pathogenesis of the rheumatoid arthritis: a systematic review. Biomed Res Int. 2016;2016:6097417. doi:10.1155/2016/6097417

17. van Hal TW, van Bon L, Radstake TR. A system out of breath: how hypoxia possibly contributes to the pathogenesis of systemic sclerosis. Int J Rheumatol. 2011;2011:824972. doi:10.1155/2011/824972

18. Pagano G, Castello G, Pallardo FV. Sjogren's syndrome-associated oxidative stress and mitochondrial dysfunction: prospects for chemoprevention trials. Free Radic Res. 2013;47:71-73. doi:10.3109/ 10715762.2012.748904

19. Perl A. Oxidative stress in the pathology and treatment of systemic lupus erythematosus. Nat Rev Rheumatol. 2013;9:674-686. doi:10.1038/nrrheum.2013.147

20. Huang $\mathrm{CC}$, Ho $\mathrm{CH}$, Chen $\mathrm{YC}$, et al. Risk of myocardial infarction after carbon monoxide poisoning: a nationwide population-based cohort study. Cardiovasc Toxicol. 2019;19:147-155. doi:10.1007/ s12012-018-9484-9

21. Huang $\mathrm{CC}$, Ho $\mathrm{CH}$, Chen $\mathrm{YC}$, et al. Increased risk for hypothyroidism associated with carbon monoxide poisoning: a nationwide population-based cohort study. Sci Rep. 2019;9:16512. doi:10.1038/ s41598-019-52844-9

22. Ball LB, Macdonald SC, Mott JA, Etzel RA. Carbon monoxide-related injury estimation using ICD-coded data: methodologic implications for public health surveillance. Arch Environ Occup Health. 2005;60:119-127. doi:10.3200/AEOH.60.3.119-127

23. England BRM. Epidemiology of, risk factors for, and possible causes of rheumatoid arthritis. 2020;2020.

24. Bengtsson AA, Rylander L, Hagmar L, Nived O, Sturfelt G. Risk factors for developing systemic lupus erythematosus: a case-control study in Southern Sweden. Rheumatology (Oxford). 2002;41:563-571. doi:10.1093/rheumatology/41.5.563

25. Cooper GS, Dooley MA, Treadwell EL, St Clair EW, Gilkeson GS. Risk factors for development of systemic lupus erythematosus: allergies, infections, and family history. $J$ Clin Epidemiol. 2002;55:982-989. doi:10.1016/S0895-4356(02)00429-8 
26. Cronje FJ, Carraway MS, Freiberger JJ, Suliman HB, Piantadosi CA. Carbon monoxide actuates $\mathrm{O}(2)$-limited heme degradation in the rat brain. Free Radic Biol Med. 2004;37:1802-1812.

27. Thom SR, Bhopale VM, Han ST, Clark JM, Hardy KR. Intravascular neutrophil activation due to carbon monoxide poisoning. Am J Respir Crit Care Med. 2006;174:1239-1248. doi:10.1164/rccm.200604-557OC

28. Xie H, Zhou F, Liu L, et al. Vitiligo: how do oxidative stress-induced autoantigens trigger autoimmunity? J Dermatol Sci. 2016;81:3-9. doi:10.1016/j.jdermsci.2015.09.003

29. Efat E. Sleep pattern changes in patients with connective tissue diseases. Egypt $J$ Chest Dis Tuberculosis. 2016;65:655-660. doi:10.1016/j.ejcdt.2016.01.015

30. Thom SR, Bhopale VM, Fisher D, Zhang J, Gimotty P. Delayed neuropathology after carbon monoxide poisoning is immune-mediated. Proc Natl Acad Sci U S A. 2004;101:13660-13665. doi:10.1073/ pnas.0405642101

31. Wang W, Li J, Chang Y, et al. Effects of immune reaction in rats after acute carbon monoxide poisoning. Undersea Hyperb Med. 2011;38:239-246.

32. Laniado-Laborin R. Smoking and chronic obstructive pulmonary disease (COPD). Parallel epidemics of the 21 century. Int J Environ Res Public Health. 2009;6:209-224. doi:10.3390/ijerph6010209

33. Pryor WA, Stone K. Oxidants in cigarette smoke. Radicals, hydrogen peroxide, peroxynitrate, and peroxynitrite. Ann N Y Acad Sci. 1993;686:12-27; discussion 27-18. doi:10.1111/j.1749-6632.1993.tb39148.x

34. Chang K, Yang SM, Kim SH, Han KH, Park SJ, Shin JI. Smoking and rheumatoid arthritis. Int J Mol Sci. 2014;15:22279-22295. doi:10.3390/ijms 151222279

35. Karabulut G, Kitapcioglu G, Inal V, et al. Cigarette smoking in primary Sjogren's syndrome: positive association only with ANA positivity. Mod Rheumatol. 2011;21:602-607. doi:10.3109/s10165011-0446-3
36. Chaudhary P, Chen X, Assassi S, et al. Cigarette smoking is not a risk factor for systemic sclerosis. Arthritis Rheum. 2011;63 (10):3098-3102. doi:10.1002/art.30492

37. Nikolova M, Liubomirova M, Iliev A, et al. Clinical significance of antinuclear antibodies, anti-neutrophil cytoplasmic antibodies and anticardiolipin antibodies in heroin abusers. Isr Med Assoc J. 2002;4:908-910.

38. Jankovic BD, Horvat J, Djordjijevic D, Ramah A, Fridman V, Spahic O. Brain-associated autoimmune features in heroin addicts: correlation to HIV infection and dementia. Int J Neurosci. 1991;58 (1-2):113-126. doi:10.3109/00207459108987188

39. Gaubitz M. Epidemiology of connective tissue disorders. Rheumatology (Oxford). 2006;45 Suppl 3:iii3-4.

40. Rasch EK, Hirsch R, Paulose-Ram R, Hochberg MC. Prevalence of rheumatoid arthritis in persons 60 years of age and older in the United States: effect of different methods of case classification. Arthritis Rheum. 2003;48:917-926. doi:10.1002/art.10897

41. Youssef WI, Tavill AS. Connective tissue diseases and the liver. $J$ Clin Gastroenterol. 2002;35:345-349. doi:10.1097/00004836200210000-00012

42. Kronbichler A, Mayer G. Renal involvement in autoimmune connective tissue diseases. BMC Med. 2013;11:95. doi:10.1186/1741-7015-11-95

43. Lundberg IE. Cardiac involvement in autoimmune myositis and mixed connective tissue disease. Lupus. 2005;14(9):708-712. doi:10.1191/0961203305lu2205oa
Clinical Epidemiology

\section{Publish your work in this journal}

Clinical Epidemiology is an international, peer-reviewed, open access, online journal focusing on disease and drug epidemiology, identification of risk factors and screening procedures to develop optimal preventative initiatives and programs. Specific topics include: diagnosis, prognosis, treatment, screening, prevention, risk factor modification,

\section{Dovepress}

systematic reviews, risk \& safety of medical interventions, epidemiology \& biostatistical methods, and evaluation of guidelines, translational medicine, health policies \& economic evaluations. The manuscript management system is completely online and includes a very quick and fair peer-review system, which is all easy to use. 